\title{
Observer-Based Synchronization of a New Hybrid Chaotic System and Its Application to Secure Communications
}

\author{
H. Bouraoui AND K. KemiH \\ EACSys-LAMEL Laboratory, Jijel University, Algeria
}

\begin{abstract}
This paper presents an interesting issue of using hybrid dynamical system to chaos synchronization and its application in secure communication using a new 5D hyperchaotic systems. The slave system is modeled by an unknown input observer, in which the unknown input issued from the hybrid system is the transmitted information. Based on the descriptor observer design, the Lyapunov stability theory and some robustness criteria, new sufficient synthesis conditions are given in terms of linear matrix inequalities that we can obtain the estimations of both the hybrid system states and the transmitted signals. Numerical examples are provided to show the performances of the proposed approach.
\end{abstract}

DOI: $10.12693 /$ APhysPolA.123.259

PACS: 05.45.Gg, 05.45.Xt

\section{Introduction}

Since Rössler first introduced the hyperchaotic dynamical system [1], many hyperchaotic systems have been proposed in the last few years $[2,3]$. Hyperchaos synchronization is one of the critical issues in nonlinear science, for its theoretical challenge and its applications in biological systems, secure communication, artificial neural networks, and so on [4]. A variety of approaches have been proposed for the synchronization of hyperchaotic systems such as linear control [5], wavelet transform [6], and so on.

In this paper, the problem addressed is the design of a synchronization scheme such that the response system can synchronize the driving system globally and its application in secure communication. In the transmission scheme, we propose a transmitter system combined of a continuous-time 5D hyperchaotic system and a discrete-time chaotic system called modified Henon. To make its structure more complex, the states of the continuous-time system are introduced in the dynamic of the discrete-time system. The receiver is composed from a continuous unknown input observer and a discrete full-order state observer. The simulation results are finally presented to visualize the satisfactory synchronization performance and the recovering of the transmitted signals.

\section{Secure communications system}

Inspired from [7], the global scheme of the proposed secure communication system is given by Fig. 1 .

The transmitter is composed of three blocks: a continuous-time hyperchaotic system, a discrete time chaotic system, and a multiplexing block.

1) Continuous-time chaotic system: Consider the following chaotic system described by:

$$
\dot{w}=A_{1} w+B_{1} s_{1}+f\left(w, s_{1}, y_{1}\right),
$$

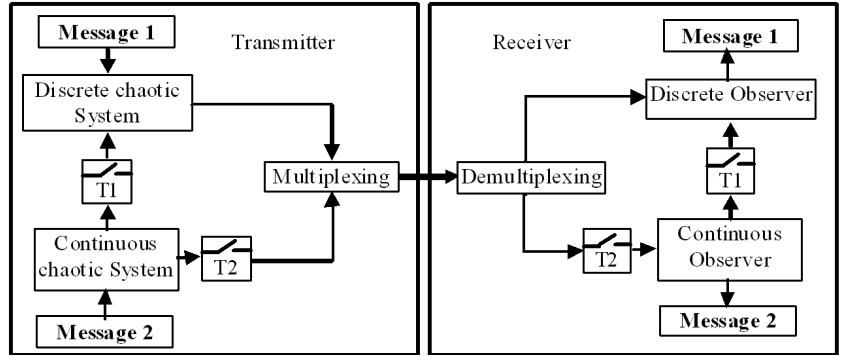

Fig. 1. Transmission chain based on a hybrid dynamical system.

$y_{1}=C_{1} w+D_{1} s_{1}$.

$w \in R^{n}, y_{1} \in R^{p}$ and $s_{1} \in R^{m}$ denote the state, output, and information signal, respectively. $A_{1}, B_{1}, C_{1}$, and $D_{1}$ are real known matrices. $f\left(w, s_{1}, y_{1}\right)$ is the nonlinear item of the system. We introduce the following notations for simplicity of the presentation:

$$
\begin{aligned}
& E=\left[\begin{array}{ll}
I_{n} & 0
\end{array}\right], \quad M=\left[\begin{array}{ll}
A_{1} & B_{1}
\end{array}\right], \quad H=\left[\begin{array}{ll}
C_{1} & D_{1}
\end{array}\right] \\
& \text { and } \boldsymbol{\varphi}=\left(\begin{array}{c}
w \\
s_{1}
\end{array}\right) .
\end{aligned}
$$

2) Discrete-time chaotic system: Consider a discrete-time chaotic system described by [8]:

$$
\begin{aligned}
& x(n+1)=A_{2} x(n)+B_{2} f_{2}(x(n))+C_{2}+B_{0} s_{2}(n), \\
& y_{2}(n)=d^{\mathrm{T}} x(n)+K f(x(n))+s_{2}(n)=\xi(n)+s_{2}(n) .
\end{aligned}
$$

$x \in R^{l}, y_{2} \in R^{q}$ and $s_{2} \in R$ denote the state, output, and information signal, respectively. $A_{2}, B_{2}$, and $C_{2}$ are real known matrices. $f_{2}(x(n))$ is the nonlinear item of the system. At the emission level, our aim is to make the structure of the discrete system more complex and to transmit the two messages. To do this, we introduce in the dynamics of discrete-time system, the states $w_{1}, w_{2}$, $w_{3}, w_{4}$, and $w_{5}$ of the continuous-time system, sampled with a rate $\mathrm{T} 1$. 


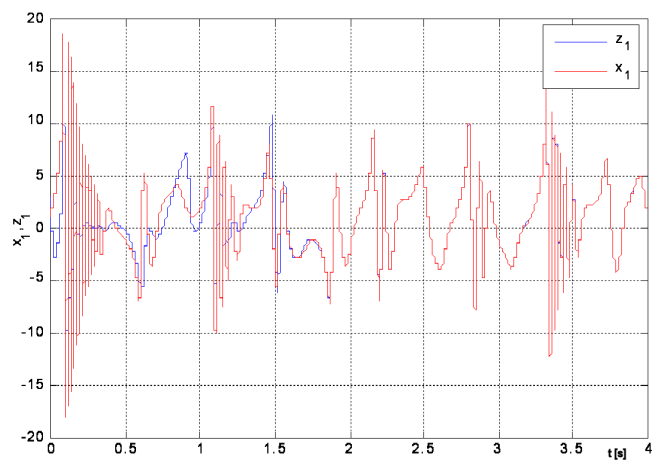

Fig. 2. Time response of $x_{1}$ and $z_{1}$.

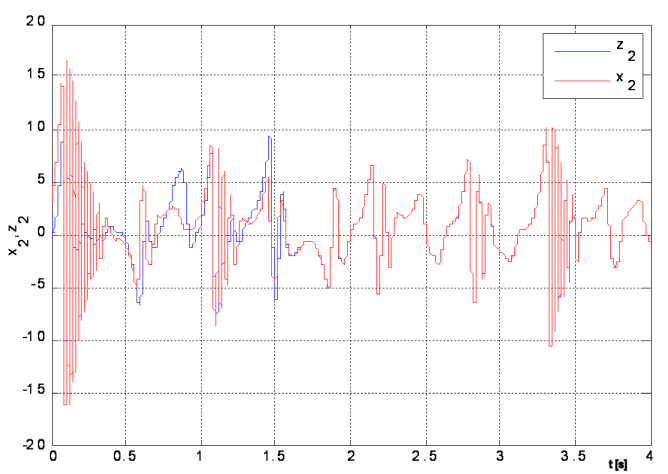

Fig. 3. Time response of $x_{2}$ and $z_{2}$.

3) Multiplexing block: each transmission cycle is composed of 10 periods T1. The signal $y_{1}$ which comes from the continuous-time system will be first sampled with a period T2, but only blocked during T1. The signal $y_{2}$ comes from the discrete-time system is sent during $9 \mathrm{~T} 1$.

The receiver is composed of three blocks: a continuous-time hyperchaotic observer, a discrete time chaotic observer and a demultiplexing block.

4) Continuous-time hyperchaotic observer: The hyperchaotic receiver system proposed is in the form [9]:

$$
\begin{aligned}
& \dot{z}=N z+L y_{1}+g\left(z, y_{1}\right), \\
& \hat{\boldsymbol{\varphi}}=z+Q y_{1},
\end{aligned}
$$

where $\hat{\varphi}$ denotes the state estimation vector of $\varphi$. $Q$ is a real matrix that verified $P E+Q H=I_{n+m}$ with $P$ is a real matrix. Matrices $N, L$ and the nonlinear vector field $g\left(z, y_{1}\right)$ should be determined such that $\hat{\boldsymbol{\varphi}}$ converges asymptotically to $\varphi$.

Consider the error vector

$$
e=\hat{\varphi}-\varphi \text {. }
$$

Substituting (4) and (2) into (5) we obtain:

$$
e=z-P E \varphi \text {. }
$$

Then, the error dynamics is

$$
\dot{e}=\dot{z}-P E \dot{\varphi} \text {. }
$$

From (2), (4) and by making use of (7), we obtain

$$
\begin{aligned}
\dot{e} & =N e+(N+F H-P M) \varphi \\
& +g\left(z, y_{1}\right)-P f\left(\boldsymbol{\varphi}, y_{1}\right)
\end{aligned}
$$

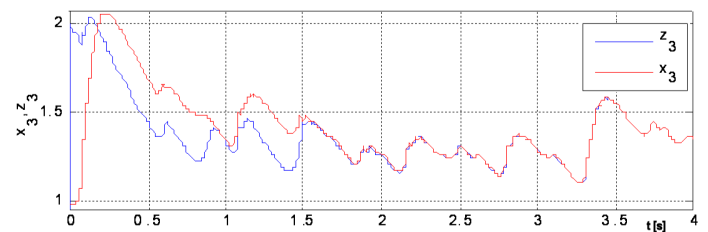

Fig. 4. Time response of $x_{3}$ and $z_{3}$.

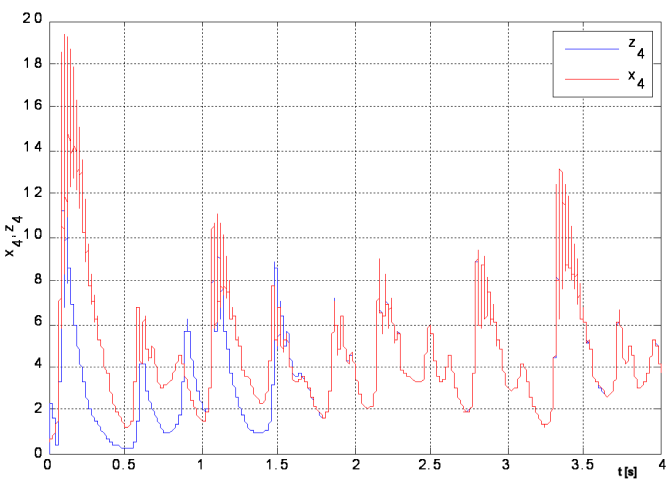

Fig. 5. Time response of $x_{4}$ and $z_{4}$.

with $F=L-N Q$. If we take

$$
N=P M-F H
$$

and $g\left(z, y_{1}\right)=\operatorname{Pf}\left(\hat{\boldsymbol{\varphi}}, y_{1}\right)=\operatorname{Pf}\left(z+Q y_{1}, y_{1}\right)$,

then the error dynamics becomes

$$
\dot{e}=N e+\operatorname{Pf}\left(\hat{\boldsymbol{\varphi}}, y_{1}\right)-\operatorname{Pf}\left(\boldsymbol{\varphi}, y_{1}\right) \text {. }
$$

In order to recover the message $z$ and $s_{1}$, the following theorem must be verified.

Theorem: The error system is asymptotically stable $\lim _{x \rightarrow \infty} e=0$ if $f(\boldsymbol{\varphi}, y)$ is assumed to be Lipschitz

$$
\left\|f\left(w, s_{1}, y_{1}\right)-f\left(z, r, y_{1}\right)\right\|<\lambda\left\|\left(\begin{array}{c}
w-z \\
s_{1}-r
\end{array}\right)\right\|
$$

with $\lambda$ a positive real scalar. D is full column rank.

The system $\left(A_{1} ; B_{1} ; C_{1} ; D_{1}\right)$ is of minimum phase. $\left(\begin{array}{cc}N^{\mathrm{T}} R+R N+\varepsilon \lambda^{2} I_{n+m} & R P \\ P^{\mathrm{T}} R & -\varepsilon I_{n+m}\end{array}\right)<0$ is solvable with $R$ a positive symmetric matrix, $\varepsilon-$ a positive number.

5) Discrete-time chaotic observer: The discrete-time receiver is described by

$$
\begin{aligned}
& \hat{x}(n+1)=A_{2} \hat{x}(n)+B_{2} f_{2}(\hat{x}(n))+C_{2} \\
& \quad+B_{0}\left[y_{2}(n)-\xi(n)\right], \\
& \xi(n)=d^{\mathrm{T}} \hat{x}(n)+K f(\hat{x}(n)) .
\end{aligned}
$$

Let $\hat{s}_{2}=\left(y_{2}-\hat{\xi}\right)$, where $\hat{x}$ denotes the state estimation vector of $x$. Matrices $B_{0}, d^{\mathrm{T}}$ and $K$ should be determined such that $\hat{s}_{2}$ converges to $s_{2}$. Defining the synchronization error: $e_{2}=\hat{x}-x$, and verifying the following theorem, we can recover the message $s_{2}$. 


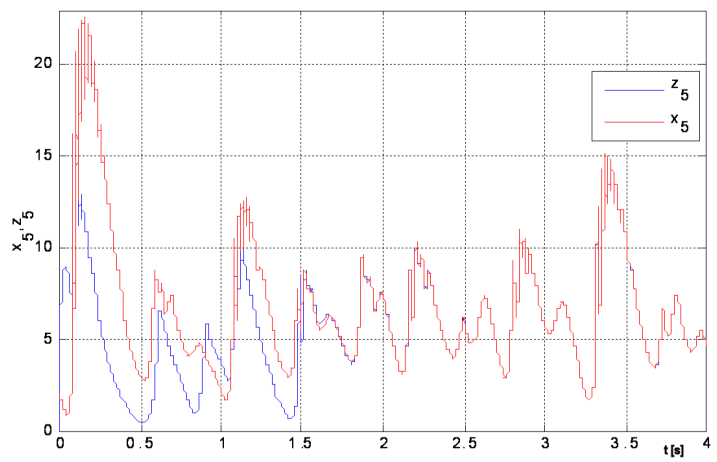

Fig. 6. Time response of $x_{5}$ and $z_{5}$.

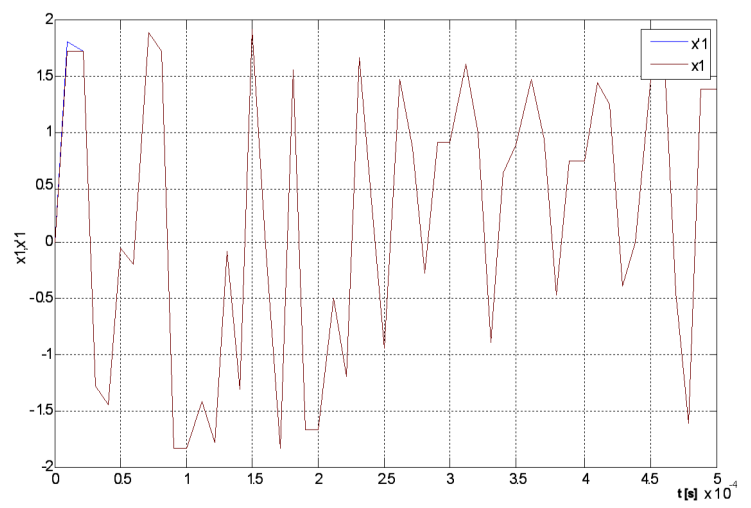

Fig. 7. Time response of $x_{1}$ and $\hat{x}_{1}$.

Theorem: The error system is asymptotically stable $\lim _{n \rightarrow \infty} e_{2}=0$ if

$$
\begin{aligned}
& B_{0}=B_{2} / K, \\
& K \neq 0,
\end{aligned}
$$

$d^{\mathrm{T}}$ satisfies: $\lambda_{i}\left(A_{2}-B_{2} d^{\mathrm{T}} / K\right)<1, i=1, \ldots, n$.

6) Demultiplexing block: First the received signal is demultiplexed on two signals $y_{1}$, and $y_{2}$. Then the signal $y_{1}$ is first memorized during a period $\mathrm{T} 2=10 \mathrm{~T} 1$, finally the signals $y_{1}, y_{2}$ are introduced, respectively, in continuous and discrete observers.

\section{Numerical results}

The fourth-order Runge-Kutta integration method is used to obtain the solutions of the continuous chaotic systems with a step size equal to 0.0001 . The initial value of the hyperchaotic system is chosen as $\left(w_{1}(0), w_{2}(0), w_{3}(0), w_{4}(0), w_{5}(0)\right)=(1,3,1,0.5,2)$. The initial value of the unknown input observer system is $\left(z_{1}(0), z_{2}(0), z_{3}(0), z_{4}(0), z_{5}(0)\right)=(3,2,2,5,4)$ and the nonlinearities of the $5 \mathrm{D}$ hyperchaotic system satisfy the Lipschitz conditions. The continuous system with hyperchaotic $5 \mathrm{D}$ system is described by [10]:

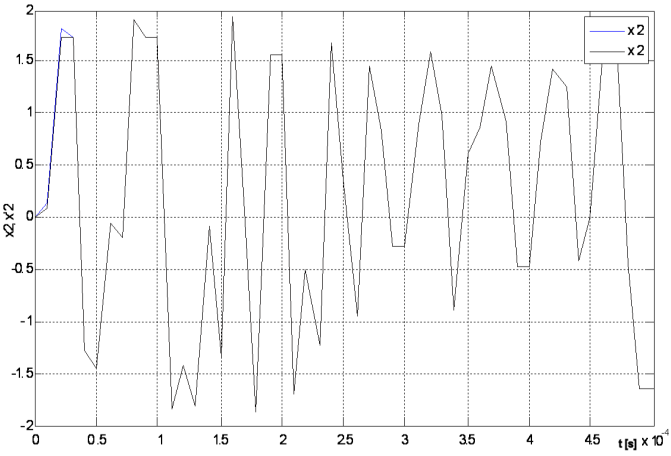

Fig. 8. Time response of $x_{2}$ and $\hat{x}_{2}$.

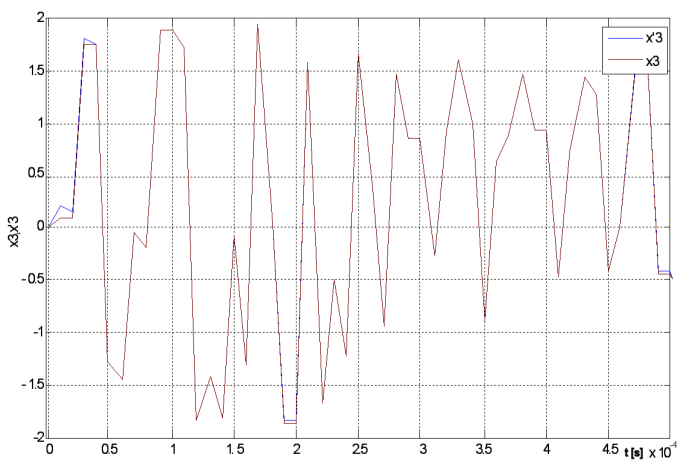

Fig. 9. Time response of $x_{3}$ and $\hat{x}_{3}$.

$$
\begin{aligned}
\dot{w}(t) & =\left(\begin{array}{ccccc}
-a_{1} & a_{1} & 0 & 0 & 0 \\
a_{2} & a_{2} & 0 & 0 & 0 \\
0 & 0 & -1 & 0 & 0 \\
0 & 0 & 0 & -a_{3} & 0 \\
-a_{5} & 0 & 0 & a_{4} & -a_{4}
\end{array}\right) w(t) \\
& +\left(\begin{array}{c}
x_{2} x_{3} x_{4} x_{5} \\
-x_{1} x_{3} x_{4} x_{5} \\
0.1 x_{1}^{2} \\
x_{1} x_{2} x_{3} x_{5} \\
x_{1} x_{2} x_{3} x_{4}
\end{array}\right)+\left(\begin{array}{c}
0 \\
30 \\
0 \\
0 \\
0
\end{array}\right) s_{1}(t),
\end{aligned}
$$$$
y_{1}=\left[\begin{array}{lllll}
0 & 1 & 0 & 0 & 0
\end{array}\right] w(t)+s_{1}(t),
$$

where $a_{1}=37, a_{2}=14.5, a_{3}=10.5, a_{4}=15$ and $a_{5}=9.5$. The first transmitted signal is $s_{1}(t)=0.5 \sin (60 \pi t)$. The discrete time chaotic system used is the modified Henon given by

$$
x(n+1)=\left[\begin{array}{ccc}
0 & 0 & -b \\
1 & 0 & 0 \\
0 & 1 & 0
\end{array}\right] x(n)+\left[\begin{array}{c}
-1 \\
0 \\
0
\end{array}\right] x_{2}^{2}(n)
$$




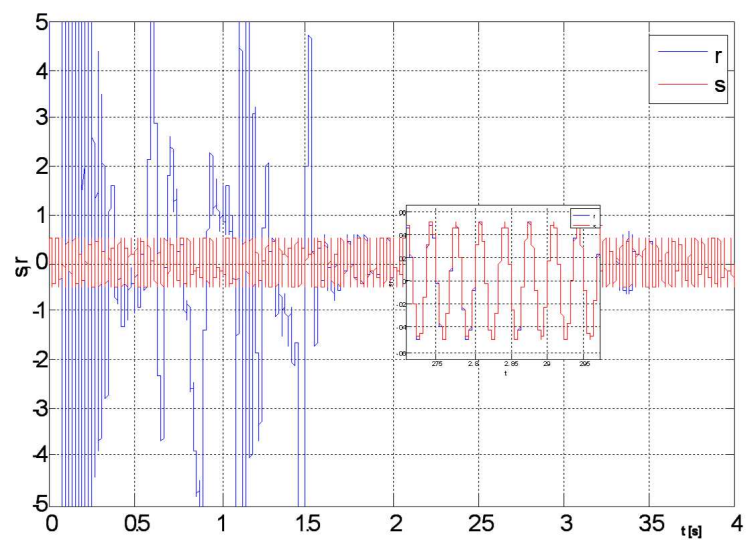

Fig. 10. Time response of $s_{1}$ and $r$. Inset is zoom for interval $t=[2.7 \div 3]$.

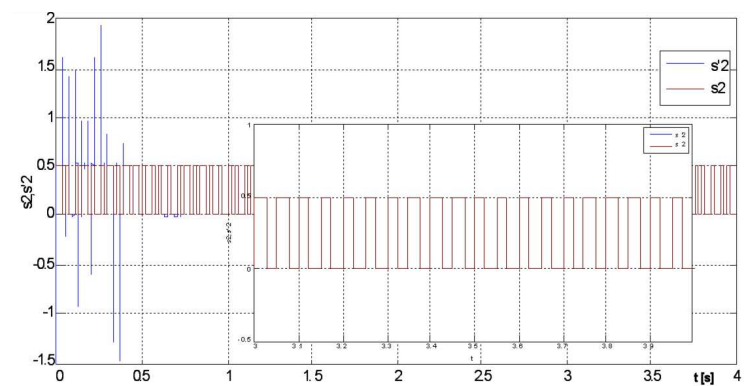

Fig. 11. Time response of $s_{2}$ and $\hat{s}_{2}$. Inset is zoom for interval $t=[3 \div 4]$.

$$
\begin{aligned}
& +\left[\begin{array}{c}
a \\
0 \\
\alpha_{1} z_{1}(n)+\alpha_{2} z_{2}(n)+\alpha_{3} z_{3}(n)+\alpha_{4} z_{4}(n)+\alpha_{5} z_{5}(n)
\end{array}\right] \\
& +\left[\begin{array}{c}
-1 \\
0 \\
0
\end{array}\right] s_{2}(n), \\
& \quad=\xi(n)+s_{2}(n),
\end{aligned}
$$

with $a=1.76, b=0.1$. The initial values are chosen as $\left(x_{1}(0), x_{2}(0), x_{3}(0)\right)=(0.1,0.1,0.1)$. The second transmitted signal $s_{2}(t)$ is a square signal with a period $T=0.05 \mathrm{~s}$.

Matrices $P, Q, N$ and $L$ of the state observer are

$$
P=\left[\begin{array}{ccccc}
1 & 0 & 0 & 0 & 0 \\
0 & 1 & 0 & 0 & 0 \\
0 & 0 & 1 & 0 & 0 \\
0 & 0 & 0 & 1 & 0 \\
0 & 0 & 0 & 0 & 1 \\
0 & -1 & 0 & 0 & 0
\end{array}\right], \quad Q=\left[\begin{array}{l}
0 \\
0 \\
0 \\
0 \\
0 \\
1
\end{array}\right], \quad L=\left[\begin{array}{c}
0 \\
30 \\
0 \\
0 \\
0 \\
-30
\end{array}\right],
$$

$$
N=\left[\begin{array}{cccccc}
-37 & -35.84 & 0 & 0 & 0 & -72.84 \\
14.50 & -66.92 & 0 & 0 & 0 & -51.42 \\
0 & 0 & -1.00 & 0 & 0 & 0 \\
0 & 7.66 & 0 & -10.50 & 0 & 7.66 \\
-9.5 & 54.19 & 0 & 15.00 & -15.00 & 54.19 \\
-14.50 & 31.80 & 0 & 0 & 0 & 16.30
\end{array}\right] .
$$

In Figs. 2-6 hyperchaotic system states and their estimations can be seen in the time interval of $[0,4] \mathrm{s}$. From Figs. 7-10 we can find that synchronization between (3) and (11) has been achieved. Figures 10 and 11 show the transmitted signals and the recovered signals. The above simulations confirm that the proposed scheme operates satisfactorily and the information signal can be asymptotically recovered.

\section{Conclusion}

This paper proposes a new secure communication scheme using the new 5D hyperchaotic system. If a master system (hybrid chaotic system) satisfies certain conditions, then the salve system can be constructed using continuous and discrete nonlinear observer to establish the synchronization and to recover the hidden messages. Simulation results have been given to demonstrate the analytical results.

\section{References}

[1] O.E. Rössler, Phys. Lett. A 71, 155 (1979)

[2] Z.L. Wang, G.Y. Wang, X.L. Bao, Chin. Phys. B 17, 3596 (2008).

[3] L. Xiao-Hua, Chin. Phys. B 18, 3304 (2009).

[4] E. Mahmoud, G.M. Mahmoud, Chaotic and Hyperchaotic Nonlinear Systems, Lambert Academic Publishing, Germany 2011.

[5] H. Wang, Z.Z. Han, Z. Mo, Comm. Nonlinear. Sci. Numerical. Sim. 15, 1910 (2010)

[6] J. Li, J. Zhou, Jiliu, Y. Wang, Y. Zhi, J. Syst. Eng. Electron. 17, 387 (2006)

[7] H. Hamiche, M. Ghanes, J.-P. Barbot, S. Djennoune, in: IEEE Int. Symp. on Communication Systems Networks and Digital Signal Processing (CSNDSP), Newcastle 2010, p. 244.

[8] Lu Jun-Guo, Xi Yu-Geng, Chin. Phys. 14, 274 (2005).

[9] M. Boutayeb, M. Darouach, H. Rafaralahy, IEEE Trans. Circuits Syst. I 49, 345 (2002)

[10] K. Kemih, H. Bouraoui, M. Ghanes, R. Remmouche, A. Senouci, Int. J. Modelling, Identif. Control 17 . 206 (2012). 